\title{
The dynamic response analysis of curved bridge under the moving load
}

\author{
$\mathrm{Li}$, Xiaodong ${ }^{\mathrm{a}}$, Ren, Jianying ${ }^{\mathrm{b}}$, Liu, Xing ${ }^{\mathrm{c}}$ \\ Shijiazhuang Tiedao University, China

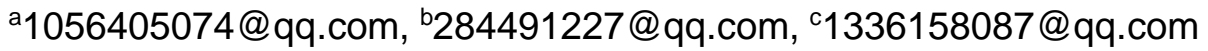

\begin{abstract}
Keywords: moving load; curved bridge; the dynamic response; finite element;
Abstract. This paper takes a four span continuous curved box girder bridge as an example. The bridge calculation model was established by finite element software ABAQUS. The calculated data is compared with the field test data to verify the model. Then the dynamic response of the bridge under different moving loads is studied. And the influence of the lateral position of the truck, axle load and centrifugal force on dynamic response of the curved bridge are analyzed. The results show that torsional effects of bridge due to eccentric loading has great influence on the curved bridge dynamic responses, and the centrifugal force obviously increases the section torque. In the elastic range, the midspan deflection and the support reaction all increase linearly with the increase of the load.
\end{abstract}

\section{Introduction}

Due to the diversity of bridge structure and the complexity and uncertainty of automobile load, the vibration analysis of highway bridge caused by vehicles becomes more complicated. Over the past few decades, the design of highway bridge mostly only for static analysis, dynamic analysis only involves the structure vibration characteristics and seismic response, the wind effect, rarely involves the analysis and study of the coupling vibration of highway curve bridge ${ }^{[1]}$. Therefore, it is necessary to study dynamic response of curved bridge under vehicle load.

The research on dynamic interaction between vehicles and bridges has been studied for a long time. A large number of scholars both at home and abroad have studied and analyzed it, and many beneficial results have been obtained ${ }^{[2-4]}$. J. Senthilvasan etc. ${ }^{[5]}$ corrected the numerical model with field test data, and analyzed the strain and deflection response of the single box double chambers curved bridge under different vehicle speed. They pointed out that the dynamic magnification factor is related to both the longitudinal position and lateral position. The results also showed that the dynamic magnification factor of strain is greater than that of deflection under the same conditions. He Xia, Yingjun Chen ${ }^{[6]}$ established the dynamic analysis model of the system. Took a system composed of four high piers, the multi-span simply supported girder bridge, and the test train as an example, the whole process of train crossing was simulated by computer, the dynamic response of the straight bridge and curved bridge under the train load is calculated. Yuan-Feng Wang and J.F. Wilson ${ }^{[7]}$ obtained analytical solutions of dynamic responses of continuous curved beams with equal spans and unequal spans for the first time by combined means of analytical solutions and finite element methods. Yumin Song etc ${ }^{[8]}$ took a small radius curve bridge on the reverse curve of a railway freight line as an example, obtained the vibration law of the curved bridge through the numerical analysis, and gave the rational driving speed of the freight train and other suggestions. Wenhui $\mathrm{Li}$ etc. ${ }^{[9]}$ adopted the space vibration analysis model of the vehicle-bridge system, simulated the whole process of a train passing through a simply supported bridge is by computer. They calculated the dynamic response of the curved bridge under the train load, the derailment coefficient of the train, the wheel load reduction rate and the train running stability index, and compared the results with the straight bridge. Most of these studies are related to Railways, and there is still less research on vehicle-bridge coupling vibration of highway bridges. The complexity and uncertainty of automobile load and the phenomenon of "bending-torsion coupling" of curve bridges make the study of highway curved bridges is very difficult. This article is based on general finite element analysis software ABAQUS, taking a four-span continuous curved bridge as an example, analyzes the dynamic response of the bridge under moving load. And compared the 
calculated data with the field test data to verify the model. Then analyzed the influence of the load lateral position, centrifugal force and vehicle load on the dynamic response of the bridge.

\section{The brief introduction to the finite element model}

Project introduction. Fig. 1 is the bridge layout of a single box double-chamber reinforced concrete curved bridge in Jiangxi province. Figures 1 (a) and 1 (b) represent the elevation and plane of the bridge respectively. The longitudinal slope of the bridge is $7 \%$, and the span is $15 \mathrm{~m}+15 \mathrm{~m}+15 \mathrm{~m}+17 \mathrm{~m}$. The average curve radius is 50 meters. The curved bridge is used for heavy truck transport, the five piers are anti-twist supports and the middle pier is fixed support. These spans from left to right are the first span, second span, third span, fourth span.

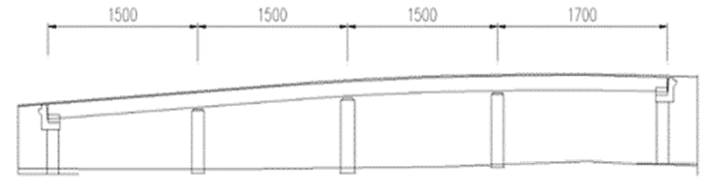

(a) Elevation of curved bridge (unit: $\mathrm{cm}$ )

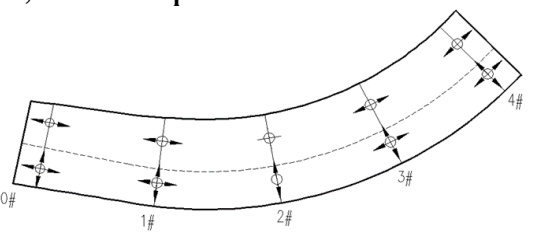

(b) Plan of curved bridge

Fig.1 Lay-out drawing of curved bridge

Fig. 2 shows the cross section diagram of the curved bridge. The roof width of the box girder is $12 \mathrm{~m}$ and the average thickness is $0.28 \mathrm{~m}$; the width of bottom is $8 \mathrm{~m}$, thickness $0.25 \mathrm{~m}$; the thickness of the web varies along the length of the girder, and the average thickness is $0.65 \mathrm{~m}$; Flange length is $2 \mathrm{~m}$.

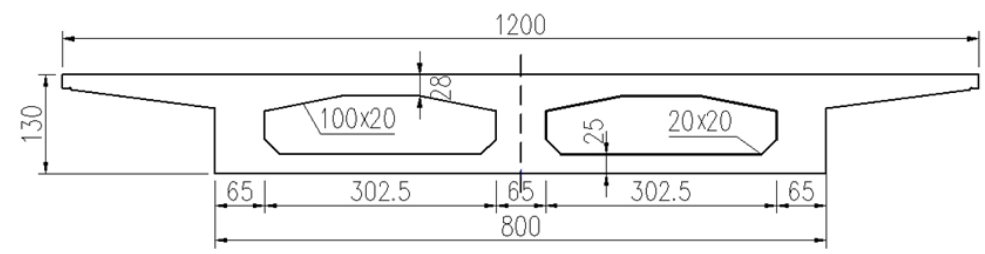

Fig. 2 The cross section of the curve box bridge

Establishment of finite element model In this paper, ABAQUS is used to establish the model of curved bridge girder. It adopts C3D8 non-decreasing integral element. And the whole model consists of 16620 solid units and 24206 nodes. The boundary constraint is applied to the node at the support according to the arrangement of the actual support. In order to simulate the actual state of the bridge, the model includes the diaphragm of the curved bridge. The beam model is shown in Fig 4.

Dong Feng Hercules heavy truck was used in the test, vehicle' model number is DFL3311AXA. Front wheel distance is $1.85 \mathrm{~m}$, rear wheel distance is $1.85 \mathrm{~m}$. The total quality is $31 \mathrm{t}$. In this model, the vehicle gravity is reduced to a pair of concentrated forces with a distance of $2 \mathrm{~m}$, centrifugal force according to formula $F_{\mathrm{c}}=\mathrm{m} v^{2} / r$ was regarded as a horizontal force along the radial direction. Simplified load diagrams are shown in Fig 3. In ABAQUS/Explicit, explicit dynamic analysis is used to calculate multiple Steps in order to simulate the whole process of the vehicle passing through the whole bridge. The dynamic response of the curved bridge in the whole process is analyzed.

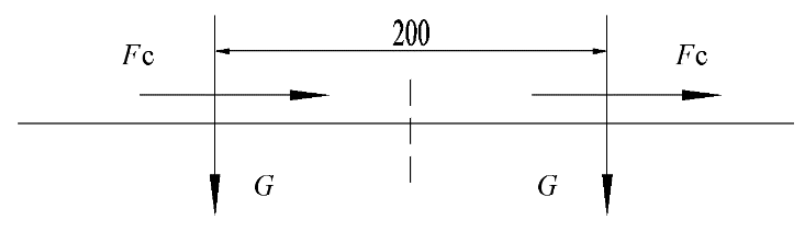

Fig.3 Simplified schema of loads

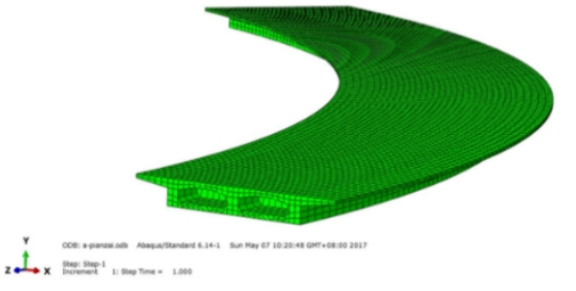

Fig.4 The finite element model of the curved bridge Correction of modulus of elasticity The reinforced concrete curved bridge adopts C50 concrete (density $\left.\quad \mathrm{c}=2500 \mathrm{~kg} / \mathrm{m}^{3}\right), V_{\mathrm{c}}=500 \mathrm{~m}^{3}$. The quality of concrete and steel bars are $M_{\mathrm{c}}=1250 \mathrm{t}$ and 
$M_{\mathrm{s}}=184.44 \mathrm{t}$ respectively. The quality of reinforcement accounts for a large proportion of the whole bridge mass, and has great influence on the rigidity of the bridge. Therefore, the influence of steel bars must be taken into account when modeling. The model is established by using the equivalent density and the equivalent stiffness.

Equivalent density calculation:

$$
\rho^{\prime}=\frac{M_{\mathrm{s}}+M_{\mathrm{c}}}{V_{\mathrm{s}}+V_{\mathrm{c}}}=\frac{184440.6+1250000}{23.5+500}=2740 \mathrm{~kg} / \mathrm{m}^{3}
$$

Where: $M_{\mathrm{s}}$ and $M_{\mathrm{c}}$ are the total mass of reinforcement and concrete; $V_{\mathrm{s}}$ and $V_{\mathrm{c}}$ are the total volume of reinforcement and concrete, respectively.

Equivalent elastic modulus calculation:

$$
\begin{aligned}
E^{\prime} & =\frac{A_{\mathrm{s}} E_{\mathrm{s}}+A_{\mathrm{c}} E_{\mathrm{c}}}{A_{\mathrm{s}}+A_{\mathrm{c}}}=\frac{A_{\mathrm{s}} E_{\mathrm{s}} L+A_{\mathrm{c}} E_{\mathrm{c}} L}{\left(A_{\mathrm{s}}+A_{\mathrm{c}}\right) L}=\frac{V_{\mathrm{s}} E_{\mathrm{s}}+V_{\mathrm{c}} E_{\mathrm{c}}}{V_{\mathrm{s}}+V_{\mathrm{c}}} \\
& =\frac{12 \times 20+500 \times 3.45}{12+500}=\frac{1965}{512}=3.838 \times 10^{10} \mathrm{~Pa}
\end{aligned}
$$

Where: $A_{\mathrm{s}}$ and $A_{\mathrm{c}}$ are the area of cross sectional reinforcement and concrete respectively; $V_{\mathrm{s}}$ is the total volume of longitudinal reinforcement; $V_{\mathrm{c}}$ is the total volume of concrete; and $L$ is the length of the beam.

Note: The materials in this model are isotropic materials.

\section{Model validation}

Fig. 5 is a field test of the bridge. The natural frequencies of the bridge, the strain distribution and the vertical deflection at the typical section of the bridge are measured. The model of the bridge is established, and the field test condition are simulated, and the results of model calculation are obtained. The calculated data is compared with the field test data to verify the model. Table 1 is the comparison between the measured values and calculated values on first and second vertical vibration frequencies of this bridge. Table 2 is comparison of the measured values and the calculated values on the third mid-span's deflections when the truck go through the curved bridge at the speed of $5 \mathrm{Km} / \mathrm{h}, 10 \mathrm{Km} / \mathrm{h}$ and $15 \mathrm{Km} / \mathrm{h}$. Table 3 shows the contrast between measured strain distribution and calculated values under static loading conditions for the tested cross section (mid-span of the third span).
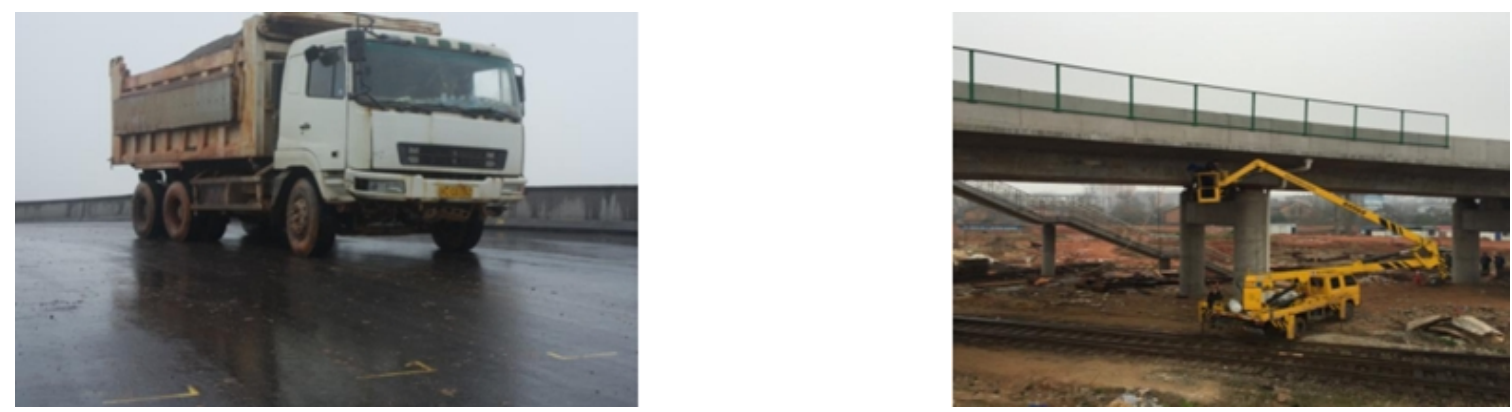

Fig.5. Field test

Tab.1 The frequency of measured values and calculated values of the curved bridge

\begin{tabular}{cccc}
\hline Order number & Measured value $(\mathrm{Hz})$ & Calculated value $(\mathrm{Hz})$ & Percentage of error $(\%)$ \\
\hline first order & 10.00 & 9.84 & 1.63 \\
second order & 12.36 & 11.50 & 9.83 \\
\hline
\end{tabular}


Tab.2 Comparison of measured values and finite element values of deflections at different speeds

\begin{tabular}{cccc}
\hline Speed $(\mathrm{km} / \mathrm{h})$ & Measured value $(\mathrm{mm})$ & Calculated value $(\mathrm{mm})$ & Percentage of error $(\%)$ \\
\hline 5 & 0.1800 & 0.1916 & 6.44 \\
10 & 0.1800 & 0.1914 & 6.33 \\
15 & 0.1700 & 0.1916 & 12.7 \\
\hline
\end{tabular}

Tab.3 Comparison of measured and finite element values of strain at dead load of the tested section

\begin{tabular}{cccc}
\hline Test location & Measured mean strain $(\quad)$ & Calculated mean strain $(\quad)$ & Percentage of error $(\%)$ \\
Under the medial flange & -8.50 & -7.10 & 16.47 \\
Box girder floor & 29.33 & 27.89 & 4.90 \\
Under the lateral flange & -8.00 & -6.93 & 13.37 \\
\hline
\end{tabular}

It can be seen from table 1 that the filed test frequency of the model is very close to that of the bridge model, and the maximum error is $9.83 \%$. It shows that the stiffness and the constraint conditions of the calculation model tend to be close to the actual situation. As can be seen from table 2, when the heavy load vehicle passes through the curved bridge at different speed, the deflection error is basically controlled below $15 \%$, and the absolute error is only $0.0216 \mathrm{~mm}$. It can be seen from table 3 that strain deviation of flange is relatively large, because the model flange plate mesh is not precise enough, but the strain error on behalf of the floor part of the overall response of box girder is very small for $4.9 \%$. Therefore, the finite element model built in this paper can simulate the actual bridge well.

\section{The influence of each parameter on the dynamic response of curved bridge}

Influence of lateral position of vehicle on dynamic response of curved bridge The vehicle load is 30t, goes through the bridge respectively along the inner $(\mathrm{e}=-3.5 \mathrm{~m})$, middle $(\mathrm{e}=0)$ and outer $(\mathrm{e}=3.5 \mathrm{~m})$ sides of the curved bridge with the speed of $10 \mathrm{~m} / \mathrm{s}$. Support forces of $2 \#$ pier on both sides, deflection on the web surface of box girder bottom of third span and the vertical deflection's distribution of third cross section when the vehicle runs to the mid-span of third span are shown in Fig 6.

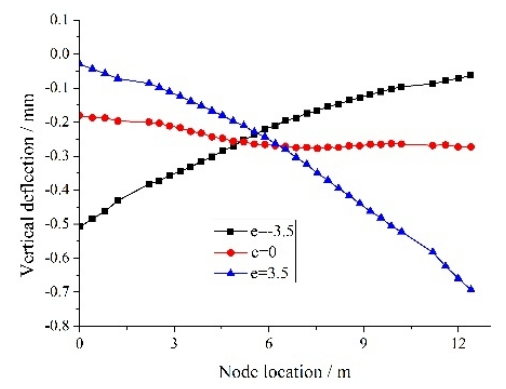

(a) Deflection of the cross section

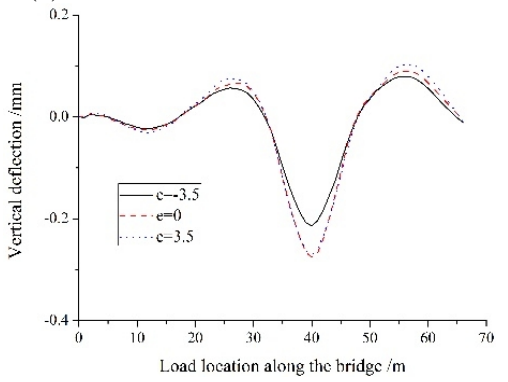

(c) Deflection at the web of the bottom of the box girder

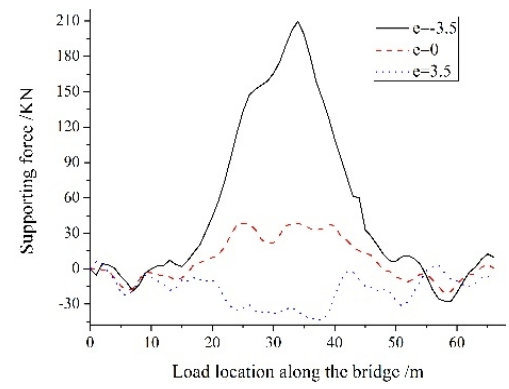

(b) Reaction of inner support

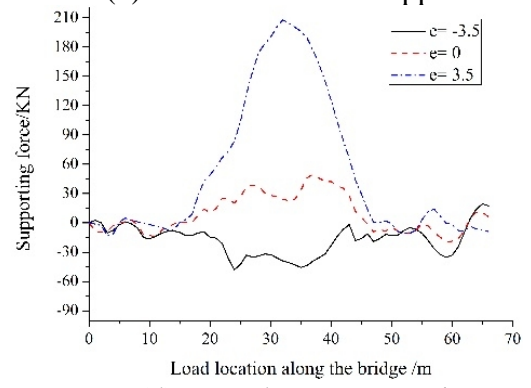

(d) Lateral support reaction

Fig.6 Influence of vehicle lateral position on dynamic response of curved bridge 
(1) It can be seen from Fig 6 (a) that the deflection and stress distribution of the curved bridge are not symmetrical under the condition of internal eccentric load and external eccentric load. Because of the effect of bending-torsion coupling, the maximum deflection and the maximum stress of the cross section under external bias load are obviously larger than those under the internal bias load; (2) From Fig 6 (b) and (d) can be seen in partial loading, lateral support is greater than the inner support force; (3) From the Fig 6 (c) can be drawn: when the vehicle running along the outside, the bottom surface has a far greater dynamic response than those in the case of the vehicle running along the inside. It shows that the torsional effect of the structure caused by eccentric load has a great influence on the dynamic response of the bridge, which agree well with the conclusion of the literature [10]. In a word, when the vehicle travels along the outside, it will cause greater dynamic response of the bridge, which is the most unfavorable condition for the vehicle running.

Effect of centrifugal force on dynamic response of curved bridge The centrifugal force of a bridge is a kind of inertia force accompanying with the moving of a vehicle in a curve, acting on the bridge in the form of horizontal forces, which is the main factor considered in the design and calculation of torsional lateral force of curved bridge ${ }^{[11]}$. General code for design of highway bridges and culverts (JTG D60-2015) clearly defined: In the design of curved bridges, the centrifugal force caused by automobile load should be calculated. The vehicle passes along the middle of the bridge at speed of $10 \mathrm{~m} / \mathrm{s}$, with the load is $30 \mathrm{t}$. The centrifugal force is $F_{\mathrm{c}}=m v^{2} / r=30000 \times 10^{2} / 50=60000 \mathrm{~N}$. The vertical deflection, lateral displacement, section torque and lateral displacement of $2 \#$ pier on the third span is shown in Fig 7.

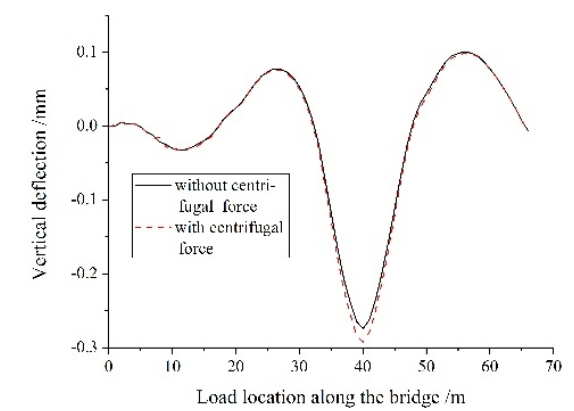

(a) Vertical deflection in midspan of the third span

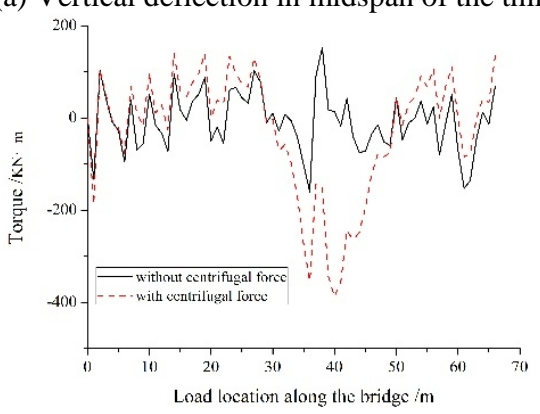

(c) Mid-span torque of third span

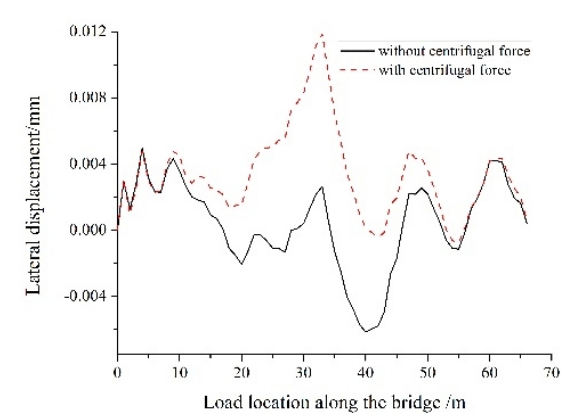

(b) Lateral displacement of 2\# lateral support

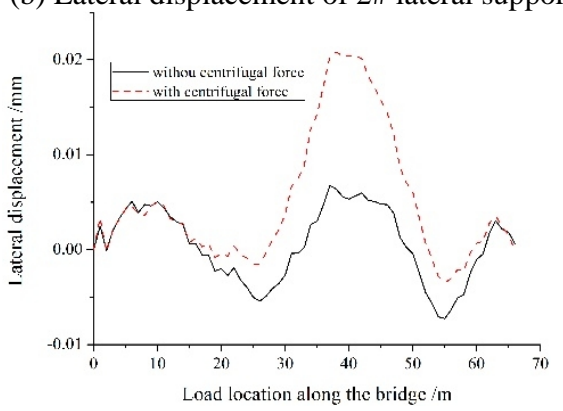

(d) Transverse displacement in midspan of third span

Fig.7 The effect of centrifugal force on dynamic response of curved bridge

(1) It can be seen from Fig 7 (a) that the centrifugal force has little effect on the vertical deflection in midspan; (2) It can be seen From Fig 7 (b), when considering the centrifugal force, the lateral displacement of the outside support of 2 \# pier is obviously increased, which shows that the centrifugal force makes the outer support causes a large lateral displacement to the outside of the curve; (3) It can be seen from Fig 7 (c) that the existence of the centrifugal force obviously increases the torque of the cross section, and will inevitably increase the torsion angle of the cross section; (4) It can be seen from Fig 7 (d) that the existence of the centrifugal force causes a larger lateral displacement of the mid-span of the third span, and makes a smaller lateral displacement to the inside of the curved bridge. To sum up, the centrifugal force has little influence on the vertical dynamic response of the bridge, but it makes the dynamic response of the section torque increase, which is consistent with the conclusions of the literature [12]. 
Influence of vehicle load on dynamic response of curved bridge The vehicle passes through the curved bridge with the load of 10t, 20t, 30t and 40t. The vertical deflection, lateral displacement and the torque of the mid-span section of third span and the supporting force and the maximum value of the outside support of $2 \#$ pier are shown in Fig 8.

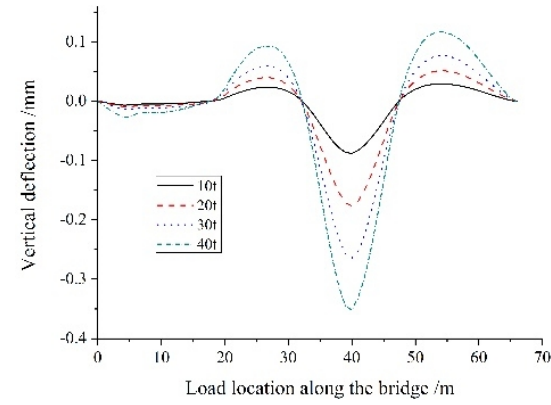

(a) Vertical deflection

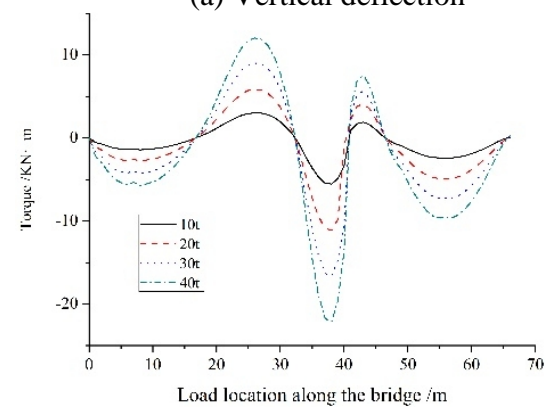

(d) Sectional torque

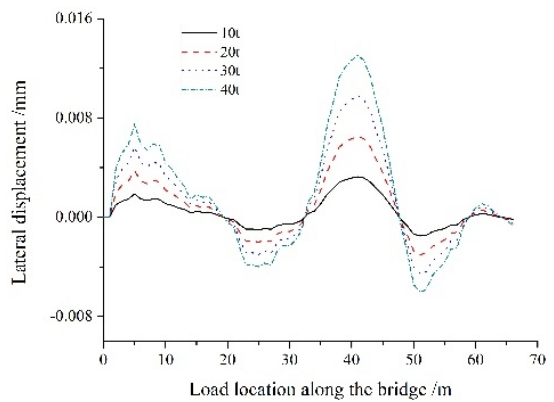

(b) Lateral displacement

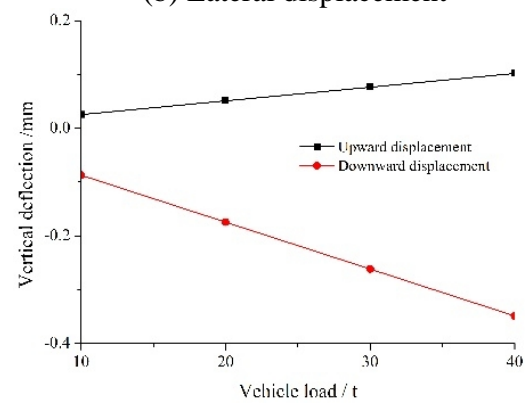

(E) Trend of deflection change

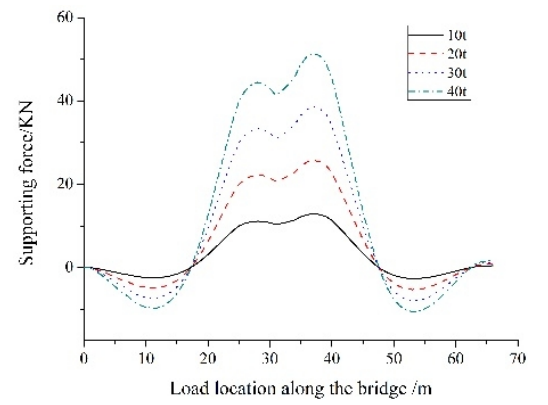

(c) Counterforce of outside support (2\#pier)

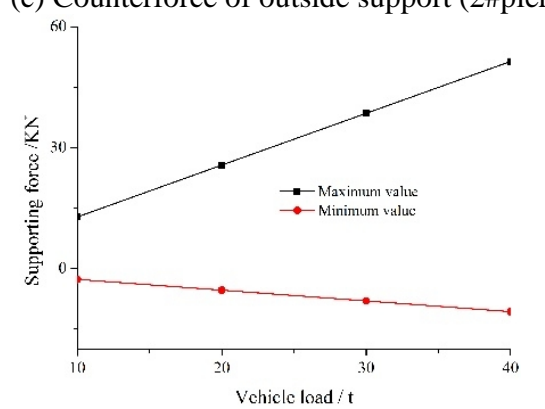

(f) Tendency of counterforce

Fig.8 The effect of vehicle weighing on dynamic response of curved bridge

Fig 8 shows that: (1) with the increase of the vehicle load, the mid-span deflection, the support reaction force and the torque on cross section of the curved bridge increase linearly. (2) The centrifugal force is proportional to the vehicle load. Under the combined influence of vehicle load and centrifugal force, the lateral displacement and the overall dynamic response of the curved bridge increase significantly.

\section{Conclusion}

A four span continuous curved box girder bridge calculation model is established by ABAQUS, and the calculation results agree well with the field test date. And the influence of the lateral position of the truck, axle load and centrifugal force on dynamic response of curved bridge are analyzed. Conclusions are as follows:

(1) The dynamic response of the bridge can be simulated better by modifying the elastic modulus of the space bridge model;

(2) The lateral driving position of the vehicle has a great influence on the dynamic response of the curved bridge. When the vehicle traveling along the lateral, lateral support is much higher than that of medial support force, and even the inside of the bearing negative reaction force. Due to the existence of bending and torsion coupling, the maximum deflection and stress of the section occur under the eccentric load condition;

(3) The centrifugal force has little influence on the vertical deflection, but it can obviously increase the section torque;

(4) In the elastic range, the mid-span deflection, the support reaction and the cross section torque increase linearly with the increase of the load.

\section{Acknowledgements}

This work was financially supported by the National Natural Science Foundation of China (11602153, 11472180). 


\section{Reference}

[1] Xueqin Cao. Dynamic analysis of bridge structures [M]. Beijing: China Railway Publishing House, 1987.

[2] Elias G. Dimitrakopoulos, Qing Zeng . A three-dimensional dynamic analysis scheme for the interaction between trains and curved railway bridges [J]. Computers and Structures, 2015, 149: 43-60.

[3] Wu S Q, Law S S. Dynamic analysis of bridge-vehicle system with uncertainties based on the finite element model [J]. Probabilistic Engineering Mechanics, 2010, 25(4): 425 - 432.

[4] Khaled M.Sennah, Xuesheng Zhang, John B.Kennedy. Impact Factors for Horizontally Curved Composite Box Girder Bridges [J]. Journal of Bridge Engineering, 2004.

[5] J. Senthilvasan, D.P. Thambiratnam, G.H. Brameld. Dynamic response of a curved bridge under moving truck load [J]. Engineering Structures, 2002, 24: 1283-1293.

[6] He Xia, Yingjun Chen. Dynamic interaction analysis of vehicle beam pier system [J]. Chinese Journal of civil engineering, 1992, 25 (2): 4-12.

[7] Yuanfeng Wang, J.F.Wilson.Dynamic response of multi span continuous curved beam under moving loads [J]. Proceedings of the civil engineering, 1999, 32 (4): 33-36.

[8] Yumin Song, Dingjun Wu, Yongjiao Hou. Analysis of the train through the small radius curve bridge dynamic interaction of reverse engineering mechanics, [J]. 2012, 29: 185-189.

[9] Wenhui Li, Kebin Xu. Dynamic curve bridge system response of railway vehicle [J]. Journal of railway engineering, 1998, 4: 50-56.

[10]Xinyi Huang, Weidong Zhuo, Hongfei Sheng. Influence of vehicle eccentricity on vehicle-bridge coupling vibration of curved beam bridge [J]. Journal of Wuhan University of Technology, 32 (9): 318-322.

[11] Yun Yang, Liemao Zhou, Yongjun Zhou. The curved bridge with high pier [M]. Beijing, China Communications Press, 2011.

[12]Xinping Zhou, Yifan Song, Shuanhai He. The highway bridge vehicle bridge coupling vibration curve numerical analysis [J]. Journal of Chang'an University, 2009, 29 (6): 43-44. 